\title{
Conservation Laws and Invariant Measures in Surjective Cellular Automata
}

\author{
Jarkko Kari If and Siamak Taati非 \\ ${ }^{1}$ Department of Mathematics, University of Turku, Finland \\ ${ }^{2}$ Department of Mathematics, University of Groningen, the Netherlands
}

We discuss a close link between two seemingly different topics studied in the cellular automata literature: additive conservation laws and invariant probability measures. We provide an elementary proof of a simple correspondence between invariant full-support Bernoulli measures and interaction-free conserved quantities in the case of onedimensional surjective cellular automata. We also discuss a generalization of this fact to Markov measures and higher-range conservation laws in arbitrary dimension. As a corollary, we show that the uniform Bernoulli measure is the only shift-invariant, full-support Markov measure that is invariant under a strongly transitive cellular automaton.

Keywords: surjective cellular automata, conservation laws, invariant measures, statistical equilibrium

\section{Introduction}

Let $\Phi: S^{\mathbb{Z}} \longrightarrow S^{\mathbb{Z}}$ be a one-dimensional reversible cellular automaton, and let $\mu: S \longrightarrow \mathbb{R}$ be a quantity that is conserved by the evolution of $\Phi$, in the sense that the average value of $\mu$ over any periodic configuration remains constant with time. Since adding a constant to $\mu$ does not change the latter condition, we may assume that $\mu$ is normalized in such a way that $\sum_{s \in S} 2^{-\mu(s)}=1$.

Suppose that the cells are initialized randomly and independently so that each state $s \in S$ appears with probability $p(s) \triangleq 2^{-\mu(s)}$. In particular, if $w=w_{1} w_{2} \cdots w_{n}$ is a word of length $n$ over $S$, the probability that $n$ consecutive cells $i+1, i+2, \ldots, i+n$ take, respectively, the states $w_{1}, w_{2}, \ldots, w_{n}$ is $p(w) \triangleq p\left(w_{1}\right) p\left(w_{2}\right) \cdots p\left(w_{n}\right)$. There is a simple argument showing that $p$ is a stationary distribution for $\Phi$; that is, after any number of iterations of $\Phi$, the state of the cells remain independent and with the same distribution $p$.

Namely, let $u$ be a word of length $l$ over $S$. If $\varphi: S^{k} \longrightarrow S$ is the local update rule of $\Phi$, then there are a finite number of words $v_{i}$ of length $k+l$ such that $\varphi\left(v_{i}\right)=u$. Let $c_{i}$ be the periodic configuration obtained by repeating $v_{i}$ on positions $\ldots,-(l+k), 0, l+k, \ldots$. The image of $c_{i}$ is a periodic configuration $e_{i}$ that is a repetition of a word of the form $x_{i} u y_{i}$ on positions $\ldots,-(l+k), 0, l+k, \ldots$ Conservation of $\mu$ implies that

$$
\mu\left(v_{i}\right)=\mu\left(x_{i}\right)+\mu(u)+\mu\left(y_{i}\right) .
$$

\footnotetext{
$\dagger$ Email: jkari@utu.fi. Research supported by the Academy of Finland Grant 131558.

‡Email: siamak.taati@gmail.com.
} 


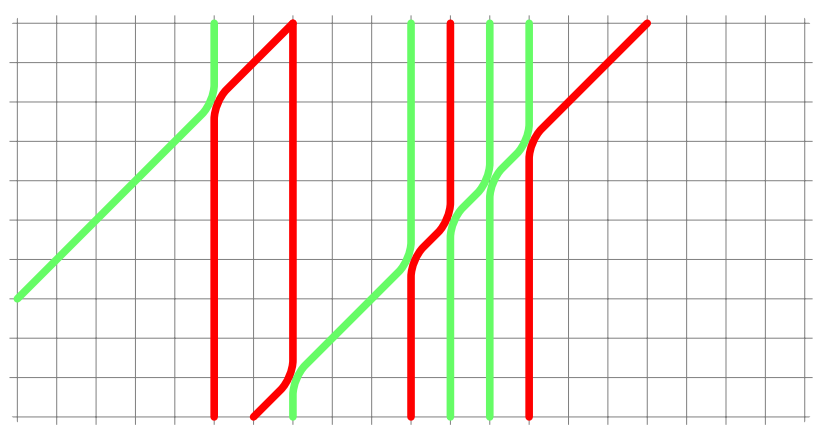

Fig. 1: Particles moving on the discrete line. Time goes downwards.

Raising 2 to the power of minus this value and summing over all $i$ we have

$$
\sum_{i} p\left(v_{i}\right)=\left[\sum_{i} p\left(x_{i}\right) p\left(y_{i}\right)\right] p(u) .
$$

But since $\Phi$ is bijective, as $i$ varies, the combination $y_{i} x_{i}$ takes all the possible values in $S^{k}$, each precisely once. Therefore, the summation part of the right-hand side adds up to 1 , and we obtain

$$
\sum_{i} p\left(v_{i}\right)=p(u)
$$

This is true for any finite word $u$, which means the joint probability distribution of the cell states remains unchanged under iterations of $\Phi$.

As an example, consider the following discrete mechanical system in one dimension, consisting of particles moving on the discrete line $\mathbb{Z}$ and interacting with each other. Each particle is either standing still, or moving to the left, with constant speed 1. Each site may contain up to two particles, one standing and one moving. Upon collision (i.e., when a moving particle meets a standing one) the moving particle stops and the standing particle starts moving. To make the model more interesting, let us assume that the particles are of two distinguishable types — red and green (Figure 1). Obviously, the total number of red or green particles is conserved with time. Furthermore, the total number of moving particles never changes. It follows from the above argument that if we choose the number and color of particles in each cell independently, according to any fixed non-vanishing probability distribution $p$, then in any future instant of time, the number and color of the particles at different sites remain independent and with the same distribution $p$.

The above observation states that for any (interaction-free) additive conserved quantity in a reversible cellular automaton, there corresponds a Bernoulli distribution that is stationary. The converse is also true: every stationary Bernoulli distribution corresponds to an additive conserved quantity. More specifically, suppose that the Bernoulli distribution with cell marginal distribution $p: S \longrightarrow(0,1]$ is stationary for $\Phi$. The claim is that the quantity $\mu(s) \triangleq-\log p(s)$ is conserved by $\Phi$.

The argument is quite similar to the previous one. Let $c_{v}$ be the periodic configuration obtained by repeating a word $v$ of length $l$ at positions $\ldots,-l, 0, l, \ldots$ Let $c_{u}=\Phi\left(c_{v}\right)$ be the image of $c_{v}$, which is 
again a repetition of a word $u$ of length $l$ at positions $\ldots,-l, 0, l, \ldots$ For an integer $n>0$, consider the word $u^{n}$. Since $\Phi$ is injective, the pre-images of $u^{n}$ under $\varphi$ are words of the form $x_{i} v^{n-2} y_{i}$. (That is, each of the pre-images has several copies of $v$ in the middle and constant-size turbulence on the borders. We have assumed that $l$ is sufficiently large.) Since $p$ is stationary, we have

$$
p\left(u^{n}\right)=\sum_{i} p\left(x_{i} v^{n-2} y_{i}\right)
$$

or

$$
p(u)^{n}=\left[\sum_{i} p\left(x_{i}\right) p\left(y_{i}\right)\right] p(v)^{n-2} .
$$

Taking the logarithm on both sides, dividing by $n$, and letting $n \longrightarrow \infty$ we obtain that

$$
\mu(u)=\mu(v) .
$$

This is the case, for any periodic configurations $c_{v}$ and $c_{u}=\Phi\left(c_{v}\right)$, which means that $\mu$ is conserved by $\Phi$.

It turns out that the above correspondence between conserved quantities and stationary Bernoulli distributions generalizes to any number of dimensions. The cellular automaton is merely required to be surjective. Finally, the conserved quantity may involve contributions from the interactions between nearby cells, in which case the corresponding probability distribution becomes a Markov measure.

This property can be proved using the variational principle of equilibrium statistical mechanics and the properties of the pre-injective factor maps on strongly irreducible shifts of finite type. It can be seen as a generalization of the balance property of the surjective cellular automata.

In the present paper we give an elementary proof of the correspondence between conserved quantities and stationary Bernoulli distributions in surjective one-dimensional cellular automata. We also state the general theorem, but the complete proof will appear elsewhere [ $\mathrm{KT}]$. In Section 5, we provide an example of how this theorem allows us to transmit results concerning conservation laws over to invariant measures.

\section{Preliminaries}

\subsection{One-dimensional cellular automata}

Let $S$ be a finite set of states. A one-dimensional cellular automaton (CA) over $S$ is a translation commuting continuous map $\Phi: S^{\mathbb{Z}} \longrightarrow S^{\mathbb{Z}}$. Equivalently, according to the Curtis-Hedlund-Lyndon theorem [Hed69], $\Phi$ is defined by a parallel application of a local rule $f: S^{2 r+1} \longrightarrow S$ at all sites, where $r$ is the neighborhood radius of $\Phi$ : for every $c \in S^{\mathbb{Z}}$ and every $i \in \mathbb{Z}$,

$$
\Phi(c)_{i}=f\left(c_{i-r}, c_{i-r+1}, \ldots, c_{i+r}\right) .
$$

Elements of $S^{\mathbb{Z}}$ are the configurations of the CA. For any word $u \in S^{l}$ of length $l \geq 1$, we denote by ${ }^{\omega} u^{\omega}$ the periodic configuration in which the word $u$ is repeated, starting in positions $\ldots,-l, 0, l, 2 l, \ldots$.

A cylinder determined by word $u$ and position $i \in \mathbb{Z}$ is the set

$$
[u]_{i} \triangleq\left\{c \in S^{\mathbb{Z}} \mid c_{i} c_{i+1} \ldots c_{i+|u|-1}=u\right\}
$$


of configurations that contain word $u$, starting in position $i$. Cylinders form a basis of the standard topology we use on $S^{\mathbb{Z}}$. Many concepts we consider are indifferent to the exact position $i$ of the cylinder, and in those cases we use the simpler notation $[u]$. This can be interpreted as any $[u]_{i}$; for example as $[u]_{0}$.

A cellular automaton $\Phi$ is injective, surjective or bijective if it is one-to-one, onto or a bijection, respectively, as a function $S^{\mathbb{Z}} \longrightarrow S^{\mathbb{Z}}$. A cellular automaton $\Phi$ is called reversible if it is bijective and its inverse is a cellular automaton. It follows from the compactness of $S^{\mathbb{Z}}$ that every bijective CA is, in fact, reversible.

Two configurations $c, e \in S^{\mathbb{Z}}$ are asymptotic if the difference set $\left\{i \in \mathbb{Z} \mid c_{i} \neq e_{i}\right\}$ is finite. A CA $\Phi$ is called pre-injective if for any asymptotic configurations $c, e$ holds that $c \neq e \Longrightarrow \Phi(c) \neq \Phi(e)$. The celebrated Garden-of-Eden theorem by E. F. Moore and J. Myhill states that $\Phi$ is surjective if and only if it is pre-injective [Moo62, Myh63]. It then directly follows that every injective CA is also surjective. We see that injectivity, bijectivity and reversibility are equivalent concepts on cellular automata, and they imply surjectivity which is equivalent to pre-injectivity.

Let $\Phi$ be defined by a radius- $r$ local rule. The local rule can be applied on finite words in a natural way, so that it defines functions $S^{k+2 r} \longrightarrow S^{k}$ for every $k$. We use the same symbol $\Phi$ also for these functions on words.

All surjective CA have the following balance property [Hed69]. Every word $u$ of length $k$ has precisely $N \triangleq|S|^{2 r}$ pre-images $v_{1}, v_{2} \ldots, v_{N} \in S^{k+2 r}$ under the function $\Phi: S^{k+2 r} \longrightarrow S^{k}$. In terms of cylinders this means that the pre-image of every cylinder $[u]_{i}$ determined by $u \in S^{k}$ is the disjoint union of the $N$ cylinders $\left[v_{1}\right]_{i-r},\left[v_{2}\right]_{i-r}, \ldots,\left[v_{N}\right]_{i-r}$ determined by words $v_{j}$ of length $k+2 r$.

\subsection{Conserved quantities}

Let $\mu: S \longrightarrow \mathbb{R}$ be an assignment of real numbers to the states. For a word $v \in S^{*}$ we define $\mu(v) \triangleq$ $\mu\left(v_{1}\right)+\mu\left(v_{2}\right)+\ldots+\mu\left(v_{k}\right)$, where $v=v_{1} v_{2} \ldots v_{k}$ and $v_{i} \in S$. Function $\mu$ is an (interaction-free) additive quantity.

A cellular automaton $\Phi: S^{\mathbb{Z}} \longrightarrow S^{\mathbb{Z}}$ conserves $\mu$ if for every $u, v \in S^{k}$ such that $\Phi\left({ }^{\omega} u^{\omega}\right)={ }^{\omega} v^{\omega}$ holds

$$
\mu(u)=\mu(v) .
$$

In other words, we require the (well defined) average value over periodic configurations to remain unchanged under the application of $\Phi$. Other equivalent characterizations exist (see e.g. [HT91, Piv02, DFR03, MBG04]). In particular, there are simple algorithms for verifying whether a given cellular automaton conserves a given additive quantity. For the proofs we present here, the above characterization using periodic configurations is most convenient.

Conserved quantities play an important role in physics, and the concept has been studied in the context of cellular automata by several authors (see e.g. [HT91, BF98, Piv02, DFR03, FG03, MBG04, Ber07, FKTar]).

\subsection{Invariant Bernoulli measures}

Let $p: S \longrightarrow \mathbb{R}$ be a probability distribution on the state set, so that $\sum_{s \in S} p(s)=1$. The Bernoulli distribution determined by $p$ is the probability distribution of a random configuration $c \in S^{\mathbb{Z}}$ if the values $c_{i}$, for $i \in \mathbb{Z}$, are chosen randomly and independently, each with distribution $p$. It is identified by a Borel probability measure $\pi$ that assigns probability $\pi([v]) \triangleq p\left(v_{1}\right) p\left(v_{2}\right) \ldots p\left(v_{k}\right)$ to each cylinder $[v]$, 
where $v=v_{1} v_{2} \cdots v_{k}$ and $v_{i} \in S$. The measure $\pi$ is translation-invariant. We only consider the case where $p(s)>0$ for all $s \in S$, which is equivalent to requiring that the Bernoulli measure $\pi$ is full-support.

A Borel measure $\pi$ is invariant (or stationary) under a cellular automaton $\Phi$ if for all cylinders $[u]$ holds $\pi([u])=\pi\left(\Phi^{-1}([u])\right)$. This means that $\pi$ is a fixed point of the mapping $\eta \mapsto \Phi(\eta)$ where $\Phi(\eta)$ is the distribution of the configuration $\Phi(c)$ if configuration $c$ is chosen randomly according to $\eta$.

The balance property of surjective CA can now be rephrased as follows: the uniform Bernoulli measure is invariant under all surjective CA.

The presence of natural invariant measures allows one to study CA as measure-preserving dynamical systems, applying results from ergodic theory (see e.g. [Wal82, Lin84, Piv09]). For instance, knowing the invariance of the uniform Bernoulli measure, one can apply Poincaré's recurrence theorem to infer that iterating a surjective CA on a uniformly random initial configuration, almost surely each finite word appearing in the initial configuration reappears infinitely many times on the same position.

\section{A correspondence of conserved quantities and invariant mea- sures on one-dimensional surjective CA}

In this section, we state and give an elementary proof for a correspondence between (interaction-free) conserved quantities and invariant, full-support Bernoulli measures on surjective, one-dimensional CA. The balance property is a special case of our theorem, so it is not surprising that the proof is similar to a standard proof of the balance property. The second part of the proof was essentially presented in [Taa09] in a more general set-up.

Theorem 1 Let $\Phi: S^{\mathbb{Z}} \longrightarrow S^{\mathbb{Z}}$ be a one-dimensional surjective cellular automaton over state set $S=$ $\left\{s_{1}, s_{2}, \ldots, s_{n}\right\}$, and let $p_{1}, p_{2}, \ldots, p_{n}$ be $n$ positive numbers such that $p_{1}+p_{2}+\ldots+p_{n}=1$. Let $\pi$ be the Bernoulli distribution on $S^{\mathbb{Z}}$ defined by $\pi\left(s_{i}\right) \triangleq p_{i}$ for all $i$, and let $\mu$ be the additive quantity defined by $\mu\left(s_{i}\right) \triangleq-\log p_{i}$. Then, $\pi$ is invariant under $\Phi$ if and only if $\Phi$ conserves $\mu$.

Proof: The base of the logarithm does not matter - we use base 2 in the proof. Let $\Phi$ be defined by a radius- $r$ local rule. Recall that $\Phi$ denotes both the CA function and the word functions $S^{k+2 r} \longrightarrow S^{k}$. Observe also the correspondence

$$
\pi([v])=2^{-\mu(v)}
$$

for all words $v \in S^{*}$.

( $\Longleftarrow$ ) Assume that $\Phi$ conserves the quantity $\mu$. Let us first prove that there are positive constants $m$ and $M$, independent of $k$, such that for all words $v \in S^{k+2 r}$ and for $u \triangleq \Phi(v) \in S^{k}$ holds

$$
m \leq \pi([v]) / \pi([u]) \leq M
$$

Indeed, let $x, y \in S^{r}$ be the words such that $\Phi\left({ }^{\omega} v^{\omega}\right)={ }^{\omega}(x u y)^{\omega}$. Then, due to the conservation of $\mu$ by $\Phi$, we have that $\mu(v)-\mu(u)=\mu(x y)$. Hence

$$
c \leq \mu(v)-\mu(u) \leq C
$$

for constants $c \triangleq 2 r \cdot \min \{\mu(s) \mid s \in S\}$ and $C \triangleq 2 r \cdot \max \{\mu(s) \mid s \in S\}$. Raising two to the negative powers of the different sides of 8 gives

$$
2^{-c} \geq \pi([v]) / \pi([u]) \geq 2^{-C},
$$




\begin{tabular}{|c|c|c|c|c|}
\hline$\alpha \in A_{i}$ & $v_{i}$ & $v_{i}$ & $v_{i}$ & $\overline{\beta \in \mathrm{B}_{i}}$ \\
\hline $\bar{u}$ & $u$ & $u$ & $u$ & $u$ \\
\hline
\end{tabular}

Fig. 2: The structure of the pre-images of word $u^{t}$.

which proves 77 .

We can now prove that $\pi$ is $\Phi$-invariant. Suppose the contrary. Then there exists a word $u \in S^{k}$ such that $\pi\left(\Phi^{-1}([u])\right)<\pi([u])$. (Indeed, if instead we have $\pi\left(\Phi^{-1}([u])\right)>\pi([u])$ for some $u \in S^{k}$, then there necessarily is another element in $S^{k}$ that has the required property.) Let $a \triangleq \pi\left(\Phi^{-1}([u])\right) / \pi([u])<1$.

The word $u$ has $N \triangleq n^{2 r}$ pre-images of length $k+2 r$, say $v_{1}, v_{2}, \ldots, v_{N}$. Let $t$ be a positive integer parameter. Consider the cylinders of the form

$$
\left[u x_{1} u x_{2} u \ldots x_{t-1} u\right]
$$

where $x_{i}$ vary over all words of length $2 r$. Let $U$ be the union of all such cylinders (for fixed t). We have that $\pi(U)=\pi([u])^{t}$. Let $V \triangleq \Phi^{-1}(U)$. Then $V$ is the union of cylinders $[w]$ over all $w \in$ $\left\{v_{1}, v_{2}, \ldots, v_{N}\right\}^{t}$, that is, over words $w$ that are concatenations of $t$ words $v_{i}$. We have that

$$
\pi(V)=\left(\pi\left(\left[v_{1}\right]\right)+\pi\left(\left[v_{2}\right]\right)+\ldots+\pi\left(\left[v_{N}\right]\right)\right)^{t}=\pi\left(\Phi^{-1}([u])\right)^{t}=(a \cdot \pi([u]))^{t}=a^{t} \cdot \pi(U) .
$$

By choosing sufficiently large $t$ we have that $\pi(V)<N \cdot m \cdot \pi(U)$, where $m$ is the constant in (7). For some $w=u x_{1} u x_{2} u \ldots x_{t-1} u$ it must then be the case that $\pi\left(\Phi^{-1}([w])\right)<N \cdot m \cdot \pi([w])$. Because $\Phi^{-1}([w])$ is the disjoint union of $N$ cylinders $[v]$ where $v$ are such that $\Phi(v)=w$, we have that for some such $v$ holds $\pi([v])<m \cdot \pi([w])$, which violates $[7]$.

$(\Longrightarrow)$ Assume that $\pi$ is invariant under $\Phi$. Let $y$ be an arbitrary periodic configuration, and let $x_{1}, x_{2}, \ldots x_{k}$ be its pre-images under $\Phi$. Note that one-dimensional surjective CA are finite-to-one, so $k$ is finite. It also follows that all $x_{i}$ are periodic. Let $p>2 r$ be a sufficiently long common period of $y$ and all $x_{i}$. We let $u \in S^{p}$ be such that $y={ }^{\omega} u^{\omega}$. For each $i$ we take $v_{i} \in S^{p}$ similarly to be the repeating period in $x_{i}$ so that $x_{i}={ }^{\omega} v_{i}^{\omega}$.

If period $p$ is chosen sufficiently long, there exist sets

$$
A_{i}, B_{i} \subseteq S^{p+r}, \text { for } i=1,2, \ldots, k,
$$

such that for every $t \geq 3$ the pre-images of the word $u^{t}$ are precisely the words

$$
\alpha v_{i}^{t-2} \beta
$$

for $i=1,2, \ldots, k$ and $\alpha \in A_{i}, \beta \in B_{i}$. See Figure 2 for an illustration. The existence of such prefix and suffix sets $A_{i}$ and $B_{i}$ for sufficiently large $p$ is a simple compactness argument.

Let $t \geq 3$ be an integer parameter, and consider the cylinder $U \triangleq\left[u^{t}\right]$ and its pre-image. We have that $\pi(U)=\pi([u])^{t}$, and by the $\Phi$-invariance of $\pi$, also

$$
\pi(U)=\pi\left(\Phi^{-1}(U)\right)=\sum_{i=1}^{k} \sum_{\alpha \in A_{i}} \sum_{\beta \in B_{i}} \pi([\alpha]) \pi\left(\left[v_{i}\right]\right)^{t-2} \pi([\beta]) .
$$


Dividing by $\pi(U)=\pi([u])^{t}$ gives

$$
1=\sum_{i}^{k} c_{i}\left(\frac{\pi\left(\left[v_{i}\right]\right)}{\pi([u])}\right)^{t},
$$

where

$$
c_{i} \triangleq \sum_{\alpha \in A_{i}} \sum_{\beta \in B_{i}} \pi([\alpha]) \pi([\beta]) \pi\left(\left[v_{i}\right]\right)^{-2}
$$

are independent of $t$.

The following obvious fact now implies that $\pi\left(\left[v_{i}\right]\right)=\pi([u])$ : if positive numbers $c_{1}, \ldots, c_{k}$ and $z_{1}, \ldots z_{k}$ satisfy

$$
1=c_{1} z_{1}^{t}+\ldots+c_{k} z_{k}^{t}
$$

for all $t=3,4, \ldots$, then necessarily all $z_{i}=1$.

It follows from $\pi\left(\left[v_{i}\right]\right)=\pi([u])$ that $\mu\left(v_{i}\right)=-\log \pi\left(\left[v_{i}\right]\right)=-\log \pi([u])=\mu(u)$. This proves the conservation of $\mu$ on periodic configurations.

\section{Generalization}

Theorem 1 can be generalized in several directions. First, suppose that rather than a Bernoulli distribution, $\pi$ is the distribution of a bi-infinite Markov chain with memory $m$, given by the transition probabilities $P(a u, u b)$ for every $a, b \in S$ and $u \in S^{m-1}$. That is, we have $\pi\left([w a u b]_{0} \mid[w a u]_{0}\right)=P(a u, u b)$ for every $a, b \in S, u \in S^{m-1}$ and $w \in S^{*}$. Assuming that all the transition probabilities $P(a u, u b)$ are non-zero, we can define an additive quantity of range $m+1$ by $\mu(a u b) \triangleq-\log P(a u, u b)$. The average value of $\mu$ on a periodic configuration $x$ is defined to be the (well defined) average value of $\mu$ on the words seen through a window of width $m+1$ that slides over $x$, and we say that $\mu$ is conserved by cellular automaton $\Phi: S^{\mathbb{Z}} \longrightarrow S^{\mathbb{Z}}$ if the average value of $\mu$ on $x$ and $\Phi(x)$ are the same for all periodic $x$. For every surjective cellular automaton $\Phi$, it can then be shown that $\pi$ is invariant under $\Phi$ if and only if $\Phi$ conserves $\mu$.

In higher dimensions, Theorem 1 remains valid as is, relating the invariance of Bernoulli distributions under surjective cellular automata and the conservation of range- 1 additive quantities.

To state the theorem in its full generality (arbitrary dimensions, arbitrary finite range) we need few definitions. Let $d \geq 1$. Let $W \subseteq \mathbb{Z}^{d}$ be a finite set. An additive quantity with interaction window $W$ is given by an assignment $\mu: S^{W} \longrightarrow \mathbb{R}$ of real numbers to patterns over $W$. If $x \in S^{\mathbb{Z}^{d}}$ is a configuration and $D \subseteq \mathbb{Z}^{d}$ a finite set, let us define $\mu_{D}(x)$ as follows: we slide the window $W$ over $x$ along the elements of $D$ and sum the values of $\mu$ over the patterns seen. That is, $\mu_{D}(x) \triangleq \sum_{i \in D} \mu\left(\left.\left(\sigma^{i} x\right)\right|_{W}\right)$, where $\sigma^{i} x$ denotes the translation of $x$ by $i$, and $\left.\left(\sigma^{i} x\right)\right|_{W}$ the restriction of $\sigma^{i} x$ to $W$. As before, we can say that a $d$-dimensional cellular automaton $\Phi: S^{\mathbb{Z}^{d}} \longrightarrow S^{\mathbb{Z}^{d}}$ conserves $\mu$ if $\mu_{D}(\Phi(x))=\mu_{D}(x)$ for every finite hypercube $D$ and every periodic configuration $x$ with fundamental domain $D$.

A Gibbs measure corresponding to $\mu$ is a Borel probability measure $\pi$ satisfying

$$
\pi\left([y]_{D}\right)=2^{-\left(\mu_{D}(y)-\mu_{D}(x)\right)} \pi\left([x]_{D}\right)
$$

for every two asymptotic configurations $x, y \in S^{\mathbb{Z}^{d}}$ and all sufficiently large finite sets $D \subseteq \mathbb{Z}^{d}$. Here, $[x]_{D}$ denotes the cylinder of all configurations that agree with $x$ on $D$. The set $D$ should be taken large 
enough so that for every $i \notin D$, the configurations $x$ and $y$ cannot be distinguished by looking through the translated window $i+W$; that is, $\left.x\right|_{i+W}=\left.y\right|_{i+W}$.

The Gibbs measures (as defined above) coincide with the full-support Markov measures (see e.g. [Pre74, Geo88]). In particular, the one-dimensional Gibbs measures are precisely the distributions of Markov chains with positive transition probabilities. However, in higher dimensions, the Gibbs measure corresponding to an additive quantity is not necessarily unique. While it can be shown that for every additive quantity there corresponds at least one Gibbs measure, distinct Gibbs measures could satisfy (9) for the same $\mu$. In statistical mechanics, Gibbs measures are used to describe the state of a system in thermal equilibrium. The multiplicity of Gibbs measures is then interpreted as the possibility of distinct equilibrium states at the same temperature (e.g., water vs. gas). See [Geo88, Pre74, KS80] for details and examples.

Theorem 2 Let $\Phi: S^{\mathbb{Z}^{d}} \longrightarrow S^{\mathbb{Z}^{d}}$ be a d-dimensional surjective cellular automaton. Let $\mu$ be an additive quantity on $S^{\mathbb{Z}^{d}}$, and let $\mathscr{G}_{\sigma}(\mu)$ denote the set of translation-invariant Gibbs measures corresponding to $\mu$. Then, the following conditions are equivalent:

a) $\Phi$ conserves $\mu$.

b) $\Phi$ maps $\mathscr{G}_{\sigma}(\mu)$ onto itself.

c) There exists an element of $\mathscr{G}_{\sigma}(\mu)$ whose $\Phi$-image is also in $\mathscr{G}_{\sigma}(\mu)$.

The proof will appear in $[\overline{\mathrm{KT}}]$.

\section{Strongly Transitive Cellular Automata}

In this section we give an example of how Theorem 2 can be used to transmit results between conservation laws research and invariant measures.

A cellular automaton $\Phi: S^{\mathbb{Z}^{d}} \longrightarrow S^{\mathbb{Z}^{d}}$ is strongly transitive if the backward orbit $\bigcup_{i=0}^{\infty} \Phi^{-i}(c)$ of every configuration $c \in S^{\mathbb{Z}^{d}}$ is dense in $S^{\mathbb{Z}^{d}}$. Equivalently, $\Phi$ is strongly transitive if for every non-empty cylinder $U \subseteq S^{\mathbb{Z}^{d}}$, the forward orbit $\bigcup_{i=0}^{\infty} \Phi^{i}(U)$ is the whole configuration space $S^{\mathbb{Z}^{d}}$. A strongly transitive CA is clearly surjective, and all positively expansive CA are strongly transitive.

Every CA has trivial conserved quantities: we call a quantity $\mu$ trivial if it assigns the same average value to all periodic configurations. For example, the interaction-free constant valuation $(\mu(s)=1$ for all $s \in S$ ) is trivial. According to (9), the uniform Bernoulli measure is the unique Gibbs measure that corresponds to trivial conserved quantities.

The following theorem states that strongly transitive cellular automata only satisfy the trivial conservation laws.

Theorem 3 ([FKTar]) Let $\Phi: S^{\mathbb{Z}^{d}} \longrightarrow S^{\mathbb{Z}^{d}}$ be a strongly transitive cellular automaton. Then $\Phi$ does not conserve any non-trivial additive quantities.

Theorems 2 and 3 now immediately give the following result:

Corollary 4 Let $\Phi: S^{\mathbb{Z}^{d}} \longrightarrow S^{\mathbb{Z}^{d}}$ be a strongly transitive cellular automaton. The uniform Bernoulli measure is the only translation-invariant Gibbs measure that is invariant under $\Phi$. 


\section{References}

[Ber07] Vincent Bernardi. Lois de conservation sur automates cellulaires. PhD thesis, Université de Provence, 2007.

[BF98] Nino Boccara and Henryk Fukś. Cellular automaton rules conserving the number of active sites. Journal of Physics A: Mathematical and General, 31(28):6007-6018, 1998.

[DFR03] Bruno Durand, Enrico Formenti, and Zsuzsanna Róka. Number conserving cellular automata I: decidability. Theoretical Computer Science, 299:523-535, 2003.

[FG03] Enrico Formenti and Aristide Grange. Number conserving cellular automata II: dynamics. Theoretical Computer Science, 304:269-290, 2003.

[FKTar] Enrico Formenti, Jarkko Kari, and Siamak Taati. On the hierarchy of conservation laws in a cellular automaton. Natural Computing, To appear.

[Geo88] Hans-Otto Georgii. Gibbs Measures and Phase Transitions. Walter de Gruyter, 1988.

[Hed69] G. A. Hedlund. Endomorphisms and automorphisms of the shift dynamical system. Mathematical System Theory, 3:320-375, 1969.

[HT91] Tetsuya Hattori and Shinji Takesue. Additive conserved quantities in discrete-time lattice dynamical systems. Physica D, 49:295-322, 1991.

[KS80] Ross Kindermann and J. Laurie Snell. Markov Random Fields and Their Applications. American Mathematical Society, 1980.

[KT] Jarkko Kari and Siamak Taati. In preparation.

[Lin84] D. A. Lind. Applications of ergodic theory and sofic systems to cellular automata. Physica D: Nonlinear Phenomena, 10(1-2), 1984.

[MBG04] Andrés Moreira, Nino Boccara, and Eric Goles. On conservative and monotone onedimensional cellular automata and their particle representation. Theoretical Computer Science, 325:285-316, 2004.

[Moo62] Edward F. Moore. Machine models of self-reproduction. In Proceedings of Symposia in Applied Mathematics, pages 17-33. AMS, 1962.

[Myh63] John Myhill. The converse of Moore's Garden-of-Eden theorem. Proceedings of the American Mathematical Society, 14:685-686, 1963.

[Piv02] Marcus Pivato. Conservation laws in cellular automata. Nonlinearity, 15:1781-1793, 2002.

[Piv09] Marcus Pivato. The ergodic theory of cellular automata. In Encyclopedia of Complexity and System Science. Springer, 2009.

[Pre74] Christopher J. Preston. Gibbs states on countable sets. Cambridge University Press, 1974. 
[Taa09] Siamak Taati. Conservation Laws in Cellular Automata. PhD thesis, University of Turku, 2009.

[Wal82] Peter Walters. An Introduction to Ergodic Theory. Springer-Verlag, 1982. 